\title{
EDITORIAL
}

For reprint orders, please contact: reprints@futuremedicine.com

\section{Integrons: epidemiological molecular markers for identifying and surveying metallo- $\beta$-lactamase genes in Gram-negative bacilli}

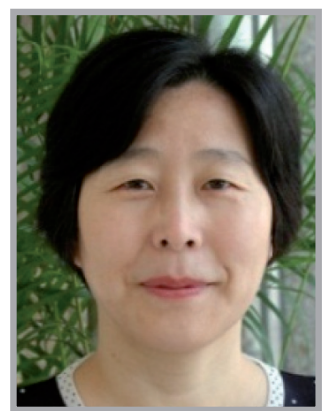

Wei-Hua Zhao*1

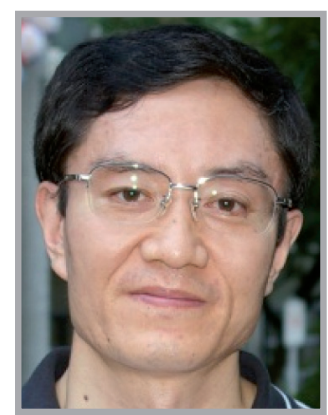

Zhi-Qing $\mathrm{Hu}^{1}$
“... analysis of the molecular diversity of integrons

may assist ... in surveying the spread and colonization of multidrug-resistant bacteria in

hospitalized patients.”
Production of $\beta$-lactamases is the major mechanism by which bacteria resist $\beta$-lactam antibiotics. Metallo- $\beta$-lactamases (MBLs) represent the greatest threat in the clinic as they are able to hydrolyze almost all $\beta$-lactams including carbapenems, and are resistant to clinically available $\beta$-lactamase inhibitors. Integrons serve as genetic apparatus that allow bacteria to capture and express gene cassettes. The MBL genes are mostly harbored by class 1 integrons whose structures display considerable diversity, suggesting different origins and acquisition events for the resistance determinants. Based on the close structural association between integrons and MBL genes, the conserved segments of the integrons may be targeted as epidemiological molecular markers for identifying and surveying MBL-producing Gram-negative bacilli.

$\beta$-lactams, including penicillins, cephems (cephalosporins and cephamycins), monobactams and carbapenems, are the most widely used antibiotics in the clinical setting. $\beta$-lactamases hydrolyze $\beta$-lactam rings and thereby inactivate their ability to bind to penicillin-binding proteins. Production of $\beta$-lactamases is the major mechanism by which bacteria resist $\beta$-lactams. Over $800 \beta$-lactamases, which are encoded by bla genes and belong to more than 30 families, have been identified in Gram-negative bacilli [1]. The increased prevalence of $\beta$-lactamases has severely reduced the clinical efficacy of the $\beta$-lactams against almost all common pathogenic bacteria.

The classification of $\beta$-lactamases is very complex owing to their enzymatic diversity. Based on molecular structures, $\beta$-lactamases are divided into four classes: A-D $[2,3]$. Classes A, C and D are serinetype enzymes possessing a serine moiety at the active site. Class B enzymes require divalent cations (usually zinc) as metal cofactors, and are thus called metallo$\beta$-lactamases. Based on substrate specificities, $\beta$-lactamases are classified into narrow-spectrum $\beta$-lactamases (penicillinases, cephalosporinases, carbenicillinases and oxacillinases, among others), extended-spectrum $\beta$-lactamases (able to hydrolyze broad-spectrum cephalosporins and monobactams) and carbapenemases (able to potently hydrolyze imipenem,

\section{KEYWORDS}

- $\beta$-lactamase $\bullet$ Gram-negative bacilli • integron

- metallo- $\beta$-lactamase

“...conserved segments of the integrons may be targeted as epidemiological molecular markers for identifying and surveying metallo- $\beta$-lactamase-producing Gram-negative bacilli.” 


\section{“Class 1 integrons in particular play a crucial role in acquiring and spreading metallo- $\beta$-lactamases."}

meropenem, panipenem and biapenem) [4,5]. In addition, based on the substrate and inhibitor profiles of $\beta$-lactamases, Bush and Jacoby proposed a functional classification in which major groupings generally correlate with molecular structures [6].

MBLs can be further divided into naturally occurring MBLs, which are usually chromosomally encoded, and acquired MBLs, which are located on transferable genetic apparatus [5]. Acquired MBLs represent the most versatile type of $\beta$-lactamases as they:

- Hydrolyze almost all $\beta$-lactams, including carbapenems, with the exception of monobactams;

- Are resistant to the clinically available $\beta$-lactamase inhibitors, such as sulbactam, clavulanate and tazobactam;

- Are supported by a mobile genetic platform $[5,7,8]$.

To date, eight acquired MBL families, including IMP, VIM, SPM, GIM, SIM, NDM, DIM and AIM, have been identified [7-14]. The IMP and VIM families are the most frequent MBLs acquired by Gram-negative bacilli $[1,5,7,8]$. In total, 42 variants of IMPs (IMP-1-42) have been assigned by the Lahey Clinic (MA, USA) [101]. IMP genes have been detected in at least 26 species of clinically important Gram-negative bacilli, such as Pseudomonas spp., Acinetobacter spp. and members of the Enterobacteriaceae family, isolated from over 24 countries/regions [7]. A total of 37 VIM variants have been assigned by the Lahey Clinic [101]; these have been detected in at least 23 species of Gram-negative bacilli from more than 40 countries/regions [8]. Compared with IMPs and VIMs, only 1-2 variants have been identified for each of the other families of acquired MBLs, including SPM, GIM, AIM, NDM, SIM and DIM [1,9-14].

Microbiological tests and molecular biological techniques are now used for the detection and identification of MBL producers. An elevated MIC of carbapenems is the first sign that a bacterial strain may be an MBL-producer. However, the detection of MBL-producing bacteria by routine testing in clinical laboratories is hindered by the fact that MBL genes do not always confer resistance. Many isolates harboring MBL genes, particularly some members of the Enterobacteriaceae family and Acinetobacter spp., are partially or completely susceptible to the carbapenems.
Some MBL-producers may be missed if susceptibility to carbapenems alone is considered for their identification. In addition, the outer membrane barrier and the active efflux system of some bacteria may also affect their susceptibility to carbapenems. Therefore, the MIC of ceftazidime (CAZ) is also commonly referenced for screening MBL-producers to avoid overlooking strains that are not resistant to carbapenems.

In addition to MBLs, there are other carbapanemases, such as Klebsiella pneumoniae carbapanemases/Guiana extended-spectrum $\beta$-lactamases (class A $\beta$-lactamases), carbapenemhydrolyzing class $\mathrm{D} \beta$-lactamases and extendedspectrum AmpC $\beta$-lactamases $[1,4,5]$. To distinguish MBL-producers from the producers of the above $\beta$-lactamases, MBL-specific inhibitors such as sodium mercaptoacetate, ethylenediaminetetraacetic acid, mercaptopropionic acid and phenanthroline are used in combination with CAZ and/or carbapenems [5,7,8]. The CAZ-sodium mercaptoacetic acid double-disk synergy test (Eiken Chemical, Tokyo, Japan) is a convenient method suitable for daily screening of MBLproducers in clinical laboratories; however, this method cannot provide information regarding the type of MBL involved. Thereafter, PCR analysis and DNA sequencing are necessary for determining the family to which an MBL belongs. By comparing the nucleotides of IMPs, however, we have noticed that it is impossible to design a pair of primers that anneal to all of the $b l a_{\mathrm{IMP}}$ genes [7]. Similarly, it is impossible to design a pair of primers that anneal to all of the $b l a_{\text {VIM }}$ genes [8]. Designing a pair of universal primers is therefore a hurdle for obtaining and sequencing PCR products of targeted MBL genes.

Integrons, first described in the late 1980s, play a critical role in the spread and expression of bla genes in bacteria [15]. Integrons serve as genetic apparatus that encode a typical integrase, allowing bacteria to capture and express genes by catalyzing a site-specific incorporation or excision of gene cassettes. Integrons can be harbored in transposons, plasmids and/or chromosomes. When integrons become associated with transposons or plasmids, forming so-called 'mobile integrons', transfer of the integron-captured genes among bacteria is readily facilitated. Of the eight classes of integrons categorized according to the divergence of integrase genes, class 1 integrons are found most commonly in pathogenic and commensal bacterial strains. Class 1 integrase is present in approximately $46 \%$ of the strains of the Enterobacteriaceae 
family isolated from both patients and animals [16], indicating that class 1 integrons are widely distributed in the environment. Class 1 integrons in particular play a crucial role in acquiring and spreading MBLs $[7,8,15]$. To this extent, a study from Japan reported that $0.5 \%$ (96/19753) of isolates of Gram-negative bacilli produced MBLs, and all of these MBL-positive strains expressed intI1, the class 1 integrase gene [17].

A typical class 1 integron consists of three regions: a $5^{\prime}$ conserved segment ( $\left.5^{\prime}-\mathrm{CS}\right)$, a variable region and a $3^{\prime}$ conserved segment (3'-CS) [7,8,15]. The 5'-CS consists of an intI site, an attI recombination site and a promoter responsible for the expression of inserted gene cassettes. The 3'-CS consists of a partially deleted qac gene ( $q a c E \Delta I$ ) fused to a sull gene, and confers resistance to antiseptics and sulfonamide. The resistance gene cassettes captured by class 1 integrons are inserted between the 5'-CS and 3'-CS. Genetic analyses have revealed that $b l a_{\mathrm{IMP}}$ and $b l a_{\mathrm{VIM}}$ genes are mostly carried by class 1 integrons and commonly coexist with one or more other resistance genes, such as aacA (the aminoglycoside resistance gene), bla ${ }_{\mathrm{OXA}}$ (the class $\mathrm{D} \beta$-lactamase gene) and cat $B$ (the chloramphenicol resistance gene) [7,8].

Integrons display considerable diversity. To date, more than 6300 entries have been added to the INTEGRALL database, which provides easy access to the DNA sequences and genetic arrangements of identified integrons [102]. At least 59 and 110 different integron structures are associated with $b l a_{\mathrm{IMP}}$ genes [7] and bla $a_{\mathrm{VIM}}$ genes [8], respectively. The diversity of the class 1 integron structures suggests different origins and acquisition events for the resistance determinants. Based on the close structural association between the class 1 integrons and MBL genes, the conserved segments of class 1 integrons may be targeted to develop novel approaches for the detection and surveillance of MBL-producing Gram-negative bacilli in a clinical setting.

\section{References}

1 Zhao WH, Hu ZQ. $\beta$-lactamases identified in clinical isolates of Pseudomonas aeruginosa. Crit. Rev. Microbiol. 36, 245-258 (2010).

2 Ambler RP. The structure of $\beta$-lactamases. Philos. Trans. R. Soc. Lond. B Biol. Sci. 289, 321-331 (1980).

3 Waley SG. $\beta$-lactamase: mechanism of action. In: The Chemistry of $\beta$-lactams. Page MI (Ed.). Chapman and Hall, London, 198-228 (1992).
MBL genes and other genetic elements between the 5'-CS and 3'-CS can be amplified using a pair of primers ( $\mathrm{F}_{\text {intI }}$-ACATGCGTGTAAATCATCGTCG annealing to the 5'-CS and $\mathrm{R}_{q u}$ ${ }_{c E_{1}} \Delta_{-}$-TGAGCCCCATACCTACAAAGC or $\mathrm{R}_{\text {sul }}^{\text {qa- }}{ }^{-}$ GTTTCCGAGAAGGTGATTGCG annealing to the 3 '-CS) under the following conditions: 30 cycles of denaturation at $95^{\circ} \mathrm{C}$ for $40 \mathrm{~s}$, annealing at $58^{\circ} \mathrm{C}$ for $60 \mathrm{~s}$ and extension at $72^{\circ} \mathrm{C}$ for $60 \mathrm{~s}$, followed by a final extension at $72^{\circ} \mathrm{C}$ for $10 \mathrm{~min}$. The resulting PCR product (2500-5000 bp) can be easily sequenced $[7,8,18]$. Based on the sequence information, it becomes possible to identify not only MBL genes (e.g., $b l a_{\mathrm{IMP}}, b l a_{\mathrm{VIM}}, b l a_{\mathrm{GIM}}$, bla${ }_{\mathrm{SIM}}, b l a_{\mathrm{NDM}}$ and $\left.b l a_{\mathrm{DIM}}\right)$, but also other adjacent resistance determinants (e.g., aacA, catB and blaOXA) simultaneously. In addition, novel resistance genes can also be discovered by this approach. It has been confirmed that the class 1 integrons can be used as an epidemiological molecular marker for the efficient identification of MBL-producers in Gram-negative bacilli [18].

As the mechanisms of integron-based capture and expression of gene cassettes are elucidated, and the cost of DNA sequencing decreases, analysis of the molecular diversity of integrons may assist in understanding the mechanisms whereby resistance is transferred between bacteria and in surveying the spread and colonization of multidrug-resistant bacteria in hospitalized patients.

\section{Financial \& competing interests disclosure}

This work was supported in part by a grant (No. 24591489) from the Ministry of Education, Culture, Sports, Science and Technology, Japan, and by a grant from Showa University Medical Foundation, Tokyo, Japan. The authors have no other relevant affliations or financial involvement with any organization or entity with a financial interest in or financial conflict with the subject matter or materials discussed in the manuscript apart from those disclosed.

No writing assistance was utilized in the production of this manuscript.

Poirel L, Pitout JD, Nordmann P.

Carbapenemases: molecular diversity and

clinical consequences. Future Microbiol. 2, 501-512 (2007).

5 Walsh TR, Toleman MA, Poirel L, Nordmann P. Metallo- $\beta$-lactamases: the quiet before the storm? Clin. Microbiol. Rev. 18, 306-325 (2005).

6 Bush K, Jacoby GA. Updated functional classification of $\beta$-lactamases. Antimicrob. Agents Chemother. 54, 969-976 (2010).

\section{"It has been confirmed that the class 1 integrons \\ can be used as an epidemiological molecular marker for the efficient identification of metallo- $\beta$-lactamase producers in Gram-negative bacilli.”}

7 Zhao WH, Hu ZQ. IMP-type metallo- $\beta$-lactamases in Gram-negative bacilli: distribution, phylogeny and association with integrons. Crit. Rev. Microbiol. 37, 214-226 (2011).

8 Zhao WH, Hu ZQ. Epidemiology and genetics of VIM-type metallo- $\beta$-lactamases in Gram-negative bacilli. Future Microbiol. 6, 317-333 (2011).

9 Toleman MA, Simm AM, Murphy TA et al. Molecular characterization of SPM-1, a novel metallo- $\beta$-lactamase isolated in Latin 
EDITORIAL Zhao \& Hu

America: report from the SENTRY antimicrobial surveillance programme. J. Antimicrob. Chemother. 50, 673-679 (2002).

10 Castanheira M, Toleman MA, Jones RN, Schmidt FJ, Walsh TR. Molecular characterization of a $\beta$-lactamase gene, bla $a_{\mathrm{GIM}-1}$, encoding a new subclass of metallo- $\beta$-lactamase. Antimicrob. Agents Chemother. 48, 4654-4661 (2004).

11 Lee K, Yum JH, Yong D et al. Novel acquired metallo- $\beta$-lactamase gene, $b_{\text {IIM-1 }}$, in a class 1 integron from Acinetobacter baumannii clinical isolates from Korea. Antimicrob. Agents Chemother. 49, 4485-4491 (2005).

12 Yong D, Toleman MA, Giske CG et al. Characterization of a new metallo$\beta$-lactamase gene, $b l a_{\mathrm{NDM}-1}$, and a novel erythromycin esterase gene carried on a unique genetic structure in Klebsiella pneumoniae sequence type 14 from India. Antimicrob. Agents Chemother. 53, 5046-5054 (2009).
13 Poirel L, Rodríguez-Martínez JM, Al Naiemi N, Debets-Ossenkopp YJ, Nordmann P. Characterization of DIM-1, an integronencoded metallo- $\beta$-lactamase from a Pseudomonas stutzeri clinical isolate in The Netherlands. Antimicrob. Agents Chemother. 54, 2420-2424 (2010).

14 Yong D, Toleman MA, Bell J et al. Genetic and biochemical characterization of an acquired subgroup B3 metallo- $\beta$-lactamase gene, $b l a_{\text {AIM-1 }}$, and its unique genetic context in Pseudomonas aeruginosa from Australia. Antimicrob. Agents Chemother. 56, 6154-6159 (2012).

15 Mazel D. Integrons: agents of bacterial evolution. Nat. Rev. Microbiol. 4, 608-620 (2006).

16 Goldstein C, Lee MD, Sanchez S et al. Incidence of class 1 and 2 integrases in clinical and commensal bacteria from livestock, companion animals, and exotics. Antimicrob. Agents Chemother. 45, 723-726 (2001).
17 Nishio H, Komatsu M, Shibata N et al. Metallo- $\beta$-lactamase-producing Gramnegative bacilli: laboratory-based surveillance in cooperation with 13 clinical laboratories in the Kinki region of Japan. J. Clin. Microbiol. 42, 5256-5263 (2004).

18 Zhao WH, Chen G, Ito R, Kimura $S$, Hu ZQ. Identification of a plasmid-borne bla $_{\mathrm{IMP}-11}$ gene in clinical isolates of Escherichia coli and Klebsiella pneumoniae. J. Med. Microbiol. 61, 246-251 (2012).

\section{- Websites}

101 Jacoby GA, Bush K. $\beta$-lactamase classification and amino acid sequences for TEM, SHV and OXA extended-spectrum and inhibitor resistant enzymes. www.lahey.org/Studies (Accessed 4 February 2013)

102 Moura A, Soares M, Pereira C, Leitão N, Henriques I, Correia A. INTEGRALL: the integron database.

http://integrall.bio.ua.pt/? (Accessed 4 February 2013) 